\title{
A Microscopic Coupled-Cluster Treatment of Electronic Correlations in Hubbard Models
}

\author{
R.F. BISHOP, Y. XIAN, AND C. ZENG \\ Department of Mathematics, UMIST (University of Manchester Institute of Science and Technology), \\ P.O. Box 88, Manchester M60 1QD, United Kingdom
}

Received August 1, 1994; accepted September 7, 1994

\begin{abstract}
We recently applied the coupled-cluster method (CCM) with considerable success to several novel quantum spin-lattice systems in the infinite bulk limit. In this article, we extend our CCM analysis to the electronic Hubbard models. In particular, based on a systematic approximation scheme within the CCM, we investigate the zero-temperature properties of Hubbard models at half-filling and with a single hole on a general bipartite lattice. Our aim was to provide the CCM framework for a systematic calculation of the properties of Hubbard models. (c) 1995 John Wiley \& Sons, Inc.
\end{abstract}

\section{Introduction}

$\mathbf{T}$ he microscopic coupled-cluster method (CCM) is widely recognized as providing one of the most powerful many-body techniques for dealing with electronic correlations in both condensed matter physics [1] and quantum chemistry [2]. Recently, we successfully applied the CCM to several novel quantum spin-lattice systems [3-5]. Using a systematic approximation scheme developed by us, we carried out calculations involving high-order many-body correlations and obtained numerical results for such physical quantities as the groundstate energy with accuracy comparable to that of the best Monte Carlo calculations available today.
For example, our best estimate for the groundstate energy per spin of the spin-1/2 Heisenberg model on the two-dimensional (2D) square lattice is $-0.6691(3)$. Furthermore, not only is our CCM analysis far less computationally intensive than are competing methods, but it also enables us to study possible quantum phase transitions of the spin-lattice systems in an extremely systematic and unbiased fashion. In this latter respect, the CCM provides an almost unique tool among highprecision methods.

In this article, we report on our progress in extending the above CCM analysis to the electronic Hubbard models on a bipartite lattice. It is well known that the Hubbard model at half-filling reduces in the strong-interaction limit to the 
spin-1/2 Heisenberg model [6]. The fact that, despite a great deal of effort, Hubbard models are still not well understood has forced many physicists to consider approaches that go beyond mean-field treatments. Examples include exact finite-size calculations, numerical simulations, and microscopic calculations based on quantum many-body theories [7]. The fundamental difficulty in tackling the Hubbard models by Monte Carlo simulations is the infamous fermion sign problem. This makes the application of well-tested many-body approaches particularly important. There have been a few applications of the CCM to the Hubbard models both at half-filling and with the further inclusion of one hole [8], based on the classical Néel model state. This approach guarantees the correct results in the large- $U$ limit (where $U$ is the on-site interaction potential). However, in the small- $U$ limit, the CCM results based on the Neel model state are not reliable. In this article, we propose a different approach within the CCM framework by employing a self-consistent mean-field wave function as a model state. The advantage of this approach lies in the fact that one is guaranteed the correct results in both the large- $U$ and small- $U$ limits.

Our aim in this article is to provide a CCM framework for a systematic investigation of the Hubbard models both at precisely half-filling and with an additional single hole, based on our previous experience for the spin-lattice systems [3-5]. The outline of the rest of this article is as follows: In the second section, following [8], we take the Néel state as the model state of the CCM and consider the full one-body correlations (singlet excitations) with arbitrary range. This one-body approximation is, in fact, closely related to the mean-field theory that is discussed in the third section. We also outline our CCM analysis using the mean-field wave function as the model state in the third section. We conclude this article in the final section with a discussion.

\section{A CCM Analysis with the Néel Model State}

We consider in this article the following Hamiltonian:

$$
\begin{aligned}
H= & -\frac{t}{2} \sum_{l} \sum_{\rho} \sum_{\sigma}\left(C_{l \sigma}^{+} C_{l+\rho \sigma}+\text { h.c. }\right) \\
& +U \sum_{l} C_{l \dagger}^{+} C_{l \uparrow} C_{l \downarrow}^{+} C_{l \downarrow},
\end{aligned}
$$

where $l$ runs over all $N$ bipartite lattice site vectors, and $\rho$ over all $z$ nearest-neighbor site vectors; $\sigma$ denotes the electron spin ( $\uparrow$ or $\downarrow$ ); $t$ is the nearestneighbor hopping parameter; and $U$ is the on-site interaction potential. The operators $C_{l \sigma}$ and $C_{l \sigma}^{+}$ are, respectively, the Hermitian-conjugate electron destruction and creation operators, which obey the usual fermionic anticommutation relations. We consider mainly positive $U$ in this article. The Hubbard model with negative $U$ can also be similarly analyzed and will be mentioned briefly at the end. At half-filling, the number of electrons, $N_{e}$, is equal to the number of sites, $N$.

\section{THE GROUND STATE}

Let us first consider the Hubbard models at half-filling $\left(N_{e}=N\right)$ in the strong coupling limit $(U \rightarrow \infty)$, where the classical Néel state is clearly a good approximation for the ground state of the Hamiltonian. The Néel state on a bipartite lattice consists of two alternating sublattices, denoted as $A$ and $B$, respectively:

$$
\left|\Phi_{N}\right\rangle \equiv \prod_{i, j} C_{i \dagger}^{+} C_{j !}^{+}|0\rangle,
$$

where, for clarity, the vector indices $\{i\}$ are used exclusively to label the position vectors of the $N / 2$ sites of the (spin up) $A$-sublattice, and the vector indices $\{j\}$, exclusively to label the position vectors of the $N / 2$ sites of the (spin down) $B$-sublattice; the state $|0\rangle$ is the fermion vacuum state. For convenience, we make the following notational changes: $C_{i \uparrow}^{+} \rightarrow a_{i \uparrow}, C_{i \uparrow} \rightarrow a_{i \uparrow}^{+}, C_{i \downarrow}^{+} \rightarrow a_{i \downarrow}^{+}, C_{i \downarrow} \rightarrow a_{i \downarrow}$ for the $A-$ sublattice and $C_{j \downarrow}^{+} \rightarrow b_{j \downarrow}^{+}, C_{j \downarrow} \rightarrow b_{j \downarrow}, C_{j \downarrow}^{+} \rightarrow b_{j \downarrow}, C_{j \downarrow} \rightarrow$ $b_{j \dagger}^{+}$for the $B$-sublattice. Hence, the Hamiltonian of Eq. (1) now becomes

$$
\begin{aligned}
H= & -t \sum_{i} \sum_{\rho}\left(a_{i \uparrow} b_{i+\rho \downarrow}\right. \\
& \left.+a_{i \downarrow}^{+} b_{i+\rho \uparrow}^{+}+\text {h.c. }\right) \\
& +U \sum_{i} a_{i \uparrow} a_{i \uparrow}^{+} a_{i \downarrow}^{+} a_{i \downarrow} \\
& +U \sum_{j} b_{j \downarrow}^{+} b_{j \downarrow} b_{j \dagger} b_{j \uparrow}^{+} .
\end{aligned}
$$

We notice that

$$
\begin{gathered}
a_{i \sigma}\left|\Phi_{N}\right\rangle=b_{j \sigma}\left|\Phi_{N}\right\rangle=0 \\
\forall i, j, \text { and } \sigma .
\end{gathered}
$$


The CCM ansatz for the exact ground ket state $\left|\Psi_{g}\right\rangle$ is

$$
\left|\Psi_{g}\right\rangle=e^{S}\left|\Phi_{N}\right\rangle, \quad S=\sum_{n=1}^{N} S_{n},
$$

where $S$ is the so-called correlation operator. It is partitioned into one-body (single excitation), twobody (double excitation), ..., up to $N$-body correlation operators, namely:

$$
\begin{aligned}
S_{1} \equiv & \sum_{i j \sigma} S_{i j}^{\sigma} a_{i \sigma}^{+} b_{j-\sigma}^{+}, \\
S_{2} \equiv & \sum_{i i^{\prime} j j^{\prime}}\left(S_{i i^{\prime}, j j^{\prime}}^{(1)} a_{i \uparrow}^{+} a_{i^{\prime} \downarrow}^{+} b_{j \downarrow}^{+} b_{j^{\prime} \uparrow}^{+}\right. \\
& +S_{i i^{\prime}, j j^{\prime}}^{(2)} a_{i \uparrow}^{+} a_{i^{\prime} \uparrow}^{+} b_{j \downarrow}^{+} b_{j^{\prime} \downarrow}^{+} \\
& \left.+S_{i i^{\prime}, j j^{\prime}}^{(3)} a_{i \downarrow}^{+} a_{i^{\prime} \downarrow}^{+} b j b^{+} b_{j^{\prime} \uparrow}^{+}\right),
\end{aligned}
$$

etc. We notice that there are two sets of coefficients in $S_{1}$ and three sets in $S_{2}$, depending on the spin configurations in each case. It is easy to prove that the correlation operators $S_{n}$ thus defined preserve the total electron number $N_{e}$ and the total electron spin along the $z$-axis. The ground-state Schrödinger equation can then be written as

$$
e^{-S} H e^{S}\left|\Phi_{N}\right\rangle=E_{g}\left|\Phi_{N}\right\rangle,
$$

where $E_{g}$ is the ground-state energy.

The most common approximation scheme in the CCM is the so-called SUBn scheme in which one retains up to $n$-body correlation operators, namely, $S \rightarrow S_{\mathrm{SUB} n}=S_{1}+S_{2}+\cdots+S_{n}$. To make a direct comparison with the mean-field model state to be discussed in the next section, we first restrict ourselves to the SUB1 (single excitation) scheme here, namely, where we replace $S \rightarrow S_{1}$ and set $S_{n}=0$ for $n>1$. The ground-state energy $E_{g}$ and the correlation coefficients (e.g., $\left\{S_{i j}^{\sigma}\right\}$ ) are determined from coupled sets of equations obtained by projection onto the Schrödinger Eq. (8). Thus, by taking the inner product of Eq. (8) with the model state itself, we obtain the energy equation

$$
E_{g}=\left\langle\Phi_{N}\left|e^{-s} H e^{s}\right| \Phi_{N}\right\rangle
$$

and by taking the inner product of Eq. (8) with states $a_{i \downarrow}^{+} b_{j \uparrow}^{+}\left|\Phi_{N}\right\rangle$ and $a_{i \uparrow}^{+} b_{j \downarrow}^{+}\left|\Phi_{N}\right\rangle$, respectively, we obtain two sets of one-body equations:

$$
\begin{aligned}
& \left\langle\Phi_{N}\left|b_{j \uparrow} a_{i \downarrow} e^{-s} H e^{S}\right| \Phi_{N}\right\rangle=0, \\
& \left\langle\Phi_{N}\left|b_{j \downarrow} a_{i \uparrow} e^{-S} H e^{S}\right| \Phi_{N}\right\rangle=0,
\end{aligned}
$$

for the corresponding coefficients $\left\{S_{i j}^{\sigma}\right\}$ with $\sigma=$ $\downarrow$ and 1 . In the above equations, the similaritytransformed Hamiltonian is given by the nested commutator series

$$
e^{-S} H e^{S}=H+[H, S]+\frac{1}{2 !}[[H, S], S]+\cdots,
$$

which actually now terminates at the second order in $S$ because $H$ contains terms of at most second order in the destruction operators $a_{i \sigma}$ and $b_{j \sigma}$.

Within the SUB1 scheme, it is quite straightforward to show that Eq. (9) becomes

$$
\frac{E_{g}}{N}=z t b_{1}
$$

Equations (10) and (11) provide a solution with $S_{i j}^{\dagger}=-S_{i j}^{\perp} \equiv b_{r}$ (with $r \equiv j-i$ ) and they both reduce in the same SUB1 approximation to

$$
t \sum_{\rho}\left(\delta_{r \rho}-\sum_{r^{\prime}} b_{r^{\prime}} b_{r-r^{\prime}+\rho}\right)+U b_{r}=0 .
$$

In Eqs. (13) and (14), $z$ is the coordination number of the lattice $(z=2$ for the $1 \mathrm{D}$ chain and $z=4$ for the 2D square lattice), and we have also employed the translational and rotational invariance.

As was done previously for the spin-lattice problems [3], one can further make a local subapproximation, namely, the SUB1-2 scheme, in which one retains only the single nearest-neighbor coefficient $b_{1}$ and sets $b_{r}=0$ for $|r|>1$. Equation (14) then reduces to

$$
t+U b_{1}-3(z-1) t b_{1}^{2}=0
$$

with the solution

$b_{1}=\frac{U}{6(z-1) t}\left(1 \pm \sqrt{1+12(z-1) t^{2} / U^{2}}\right)$.

We substitute the physical solution (with the negative sign of the square root) into Eq. (13) and obtain $E_{g}$ as a function of $U$ in the SUB1-2 scheme:

$\frac{E_{g}}{N}=\frac{z U}{6(z-1)}\left(1-\sqrt{1+12(z-1) t^{2} / U^{2}}\right)$.

This result agrees with that of previous CCM calculations [8] performed at this level.

More interestingly, we can also solve the full SUB1 Eq. (14) by a sublattice Fourier transformation technique similar to that used in the SUB2 scheme for the spin-lattice systems [3]. The physical solution is easily obtained after the Fourier transformation as

$$
b_{1}=\frac{1}{k} \frac{2}{N} \sum_{\mathbf{q} \in \mathcal{M}}\left(1-\sqrt{1+k^{2} \gamma_{\mathbf{q}}^{2}}\right),
$$

where we define

$$
k \equiv \frac{2 z t}{U}, \quad \gamma_{\mathbf{q}} \equiv \frac{1}{z} \sum_{\rho} \exp (i \mathbf{q} \cdot \rho)
$$


and where the summation $\sum_{\mathbf{q} \in \mathcal{M}}$ is over the reciprocal lattice of the $A$ - or $B$-sublattice (i.e., the magnetic zone $\mathcal{M})$, which, in the infinite bulk limit $(N \rightarrow \infty)$, can be written as an integral,

$$
\begin{aligned}
\sum_{\mathbf{q} \in \mathcal{M}} & \equiv N \int_{\mathcal{M}} \frac{d \mathbf{q}}{(2 \pi)^{d}} \\
& =\frac{N}{2} \int_{-\pi}^{\pi} \cdots \int_{-\pi}^{\pi} \frac{d q_{1}}{2 \pi} \cdots \frac{d q_{d}}{2 \pi},
\end{aligned}
$$

for a system defined on a $d$-dimensional bipartite lattice. In the above second equality, we used the symmetry of $\gamma_{\mathbf{q}}$ to write the integral over the entire Brillouin zone because all integrands in this article are functions of $\gamma_{\mathbf{q}}^{2}$. The ground-state energy in the full SUB1 scheme is then given by

$$
\frac{E_{g}}{N}=\frac{U}{2} \frac{2}{N} \sum_{\mathbf{q} \in \mathcal{M}}\left(1-\sqrt{1+k^{2} \gamma_{\mathbf{q}}^{2}}\right) .
$$

It is easy to see that the ground-state energy from both the SUB1-2 and full SUB1 schemes produces the correct large- $U$ limit, namely:

$$
\frac{E_{g}}{N} \rightarrow-\frac{z t^{2}}{U}, \quad \frac{t}{U} \rightarrow 0 .
$$

We note that there are no terminating points for the Hubbard models in the solution of the full SUB1 equation, whereas in the SUB2 approximation for the anisotropic Heisenberg spin-lattice systems [3] there was a terminating point in the solution. These terminating points were found to correspond to the quantum phase transition point between Isinglike and $X Y$-like phases. We therefore conclude that there is no phase transition for the Hubbard model at half-filling within this SUB1 scheme.

\section{THE EXCITED STATES}

The Hubbard model at half-filling plus a single hole (i.e., $N_{e}=N-1$ ) can be conveniently considered as the charge excitation state at half-filling. We can then use the usual CCM formalism for the excited states to study the single-hole problem for the Hubbard models in a systematic fashion. The CCM formalism for the excited states has been successfully applied to the spin-lattice systems to obtain the spin-wave spectra by us [3]. In this formalism, first developed by Emrich within the context of applications to finite nuclei [9], the excited state $\left|\Psi_{e}\right\rangle$ takes the form

$$
\left|\Psi_{e}\right\rangle=X\left|\Psi_{g}\right\rangle=X e^{S}|\Phi\rangle,
$$

where $|\Phi\rangle$ is the model state, $S$ is the ground-state correlation operator as determined above, and $X$, which appears linearly in Eq. (23), is the excitation correlation operator consisting only of creation operators. It is thus constructed in a similar fashion to the operator $S$. By combining the Schrödinger equations of the excited and ground states, one easily derives

$e^{-S}[H, X] e^{S}|\Phi\rangle=\omega|\Phi\rangle, \quad \omega \equiv E_{e}-E_{g}$,

where $\omega$ is the difference between the energies of the excited and ground states.

For the present case, the Néel state is again chosen as the model state, $|\Phi\rangle=\left|\Phi_{N}\right\rangle$. One of the charge excitation operators is then defined as $X=\sum_{n} X_{n}$ with

$$
\begin{aligned}
& X_{1}=\sum_{i} \chi_{i} a_{i \uparrow}^{+}, \\
& X_{2}=\sum_{i, i^{\prime}, j}\left(\chi_{i i^{\prime} j}^{(1)} a_{i \uparrow}^{+} a_{i^{\prime} \uparrow}^{+} b_{j \downarrow}^{+}+\chi_{i i^{\prime} j}^{(2)} a_{i \uparrow}^{+} a_{i^{\prime} \backslash}^{+} b_{j \dagger}^{+}\right),
\end{aligned}
$$

etc. The other three charge excitation operators can be defined in a similar fashion.

Again, as a demonstration, we consider only the simple so-called SUB $(1,1)$ approximation scheme here, wherein we retain only $S_{1}$ in $S$ and $X_{1}$ in $X$. The equation for the coefficient $\left\{\chi_{i}\right\}$ is obtained by the projection of the Schrödinger Eq. (24) with the state $a_{i \uparrow}^{+}\left|\Phi_{N}\right\rangle$. After the Fourier transformation, it is easy to obtain the excitation spectrum (or the single-hole spectrum) as a function of the lattice momentum $\mathbf{q}$ as follows:

$$
\begin{gathered}
\omega_{\mathbf{q}}=\frac{U}{2} \sqrt{1+k^{2} \gamma_{\mathbf{q}}^{2}}=\sqrt{U^{2} / 4+\epsilon_{\mathbf{q}}^{2}}, \\
\epsilon_{\mathbf{q}} \equiv-z t \gamma_{\mathbf{q}},
\end{gathered}
$$

where the definition of $k$ in Eq. (19) is used and $\epsilon_{\mathbf{q}}$ is the free-particle spectrum. The spectrum $\omega_{\mathbf{q}}$ has a minimum on the 2D square lattice for $\pm q_{x} \pm q_{y}=$ $\pi$, namely, on the boundary of its magnetic zone $\mathcal{M}$. We shall compare this result below with the corresponding result from mean-field theory.

\section{The Mean-field ccM Analysis}

The above CCM analysis based on the Néel state produces the correct large- $U$ limit even in the SUB1 scheme. However, its results in the small- $U$ limit are not reliable. Here, we explore the possibility of a CCM analysis that produces correct results in both 
the large- $U$ and small- $U$ limits by employing the mean-field wave function as our new model state. We first discuss the mean-field theory and then we outline the CCM analysis using the mean-field wave function as the model state.

\section{THE MEAN-FIELD THEORY}

The mean-field theory for the Hubbard model at half-filling has been investigated by a number of authors [10]. As is well known, a mean-field theory includes only one-body correlations. To make direct comparison with the CCM analysis discussed above, we discuss the mean-field theory that includes the one-body correlations with respect to the Néel state. We therefore write our mean-field wave function $\left|\Phi_{M F}\right\rangle$ as

$$
\left|\Phi_{M F}\right\rangle=A_{0} \exp \sum_{\mathbf{q} \in \mathcal{M}} \tan \theta_{\mathbf{q}}\left(a_{\mathbf{q} \mathbf{l}}^{+} b_{-\mathbf{q} \downarrow}^{+}-a_{\mathbf{q} \mathbf{l}}^{+} b_{-\mathbf{q} \mathbf{q}}^{+}\right)\left|\Phi_{N}\right\rangle,
$$

where $A_{0}$ is the normalization factor, $\sum_{\mathbf{q} \in \mathcal{M}}$ is as given by Eq. (20), $\left|\Phi_{N}\right\rangle$ is the Néel state as defined in Eq. (2), and we have performed the usual sublattice Fourier transformations for the fermion operators by

$$
a_{\mathbf{q} \sigma}=\sqrt{\frac{2}{N}} \sum_{i=1}^{N / 2} e^{-i \mathbf{q} \cdot r_{i}} a_{i \sigma}, \quad \sigma=\uparrow, \downarrow,
$$

etc., with $\mathbf{r}_{i}$ the position vector of the $i$ th lattice site on the $A$-sublattice. In the mean-field wave function $\left|\Phi_{M F}\right\rangle, \theta_{\mathbf{q}}$ is the parameter that is now chosen to diagonalize the mean-field Hamiltonian $H_{M F}$ that contains only up to quadratic terms in the fermion operators. This $H_{M F}$ is obtained by the usual decoupling approximation for the interaction terms, namely:

$$
\begin{aligned}
a_{i \uparrow} a_{i \uparrow}^{+} a_{i \downarrow}^{+} a_{i \downarrow} \approx & -\left\langle a_{i \uparrow} a_{i \uparrow}^{+}\right\rangle\left\langle a_{i \downarrow}^{+} a_{i \downarrow}\right\rangle \\
& +\left\langle a_{i \downarrow}^{+} a_{i \downarrow}\right\rangle a_{i \uparrow} a_{i \uparrow}^{+}+\left\langle a_{i \uparrow} a_{i \uparrow}^{+}\right\rangle a_{i \downarrow}^{+} a_{i \downarrow},
\end{aligned}
$$

etc., where the expectation value $\langle\ldots\rangle$ is taken with respect to the mean-field wave function of Eq. (28). One therefore obtains a self-consistency equation for $\theta_{\mathbf{q}}$ as

$$
\tan \left(2 \theta_{\mathbf{q}}\right)=\frac{\epsilon_{\mathbf{q}}}{\Delta},
$$

with the same $\epsilon_{\mathbf{q}}$ as in Eq. (27) and where the gap parameter $\Delta$ is defined in terms of the parameter $\left\{\theta_{\mathbf{q}}\right\}$ via

$$
\Delta \equiv \frac{1}{2} U\left(n_{\uparrow}-n_{\ddagger}\right),
$$

with $n_{\uparrow} \equiv\left\langle a_{i \uparrow} a_{i \uparrow}^{+}\right\rangle=\left\langle b_{j \uparrow} b_{j \dagger}^{+}\right\rangle$and $n_{\downarrow} \equiv\left\langle a_{i \downarrow}^{+} a_{i \downarrow}\right\rangle=$ $\left\langle b_{j l}^{+} b_{j l}\right\rangle$.

The diagonalized mean-field Hamiltonian can then be written as

$$
H_{M F}=E_{0}+\sum_{\mathbf{q} \in \mathcal{M}} \sum_{\sigma} E_{\mathbf{q}}\left(\alpha_{\mathbf{q} \sigma}^{+} \alpha_{\mathbf{q} \sigma}+\beta_{\mathbf{q} \sigma}^{+} \beta_{\mathbf{q} \sigma}\right),
$$

where $E_{\mathrm{q}}$ and $E_{0}$ are, respectively, the excitation spectrum and the mean-field ground-state energy:

$$
\begin{aligned}
E_{\mathbf{q}} & \equiv \sqrt{\epsilon_{\mathbf{q}}^{2}+\Delta^{2}}, \\
\frac{E_{0}}{N} & \equiv \frac{U}{4}+\frac{\Delta^{2}}{U}-\frac{2}{N} \sum_{\mathbf{q} \in \mathcal{M}} E_{\mathbf{q}},
\end{aligned}
$$

and where the new fermion operators $\alpha_{\mathrm{q} \sigma}$ and $\beta_{\mathbf{q} \sigma}$ are related to the old fermion operators by the usual Bogoliubov transformation:

$$
\begin{aligned}
& a_{\mathbf{q} \uparrow}=\cos \theta_{\mathbf{q}} \alpha_{\mathbf{q} \uparrow}+\sin \theta_{\mathbf{q}} \beta_{-\mathbf{q} \downarrow} ; \\
& b_{\mathbf{q} \downarrow}=-\sin \theta_{\mathbf{q}} \alpha_{-\mathbf{q} \uparrow}+\cos \theta_{\mathbf{q}} \beta_{\mathbf{q} \downarrow}, \\
& a_{\mathbf{q} \downarrow}=\cos \theta_{\mathbf{q}} \alpha_{\mathbf{q} \downarrow}+\sin \theta_{\mathbf{q}} \beta_{-\mathbf{q} \uparrow} \\
& b_{\mathbf{q} \uparrow}=\sin \theta_{\mathbf{q}} \alpha_{-\mathbf{q} \downarrow}-\cos \theta_{\mathbf{q}} \beta_{\mathbf{q} \uparrow},
\end{aligned}
$$

etc. The mean-field wave function $\left|\Phi_{M F}\right\rangle$ is the vacuum state for the quasi-particle operators $\alpha_{\mathbf{q} \sigma}$ and $\beta_{\mathbf{q} \sigma}$, namely:

$$
\alpha_{\mathbf{q} \sigma}\left|\Phi_{M F}\right\rangle=\beta_{\mathbf{q} \sigma}\left|\Phi_{M F}\right\rangle=0 .
$$

Using these quasi-particle operators, it is easy to derive

$$
n_{\uparrow}=\frac{2}{N} \sum_{\mathbf{q} \in \mathcal{M}} \cos ^{2} \theta_{\mathbf{q}}, \quad n_{l}=\frac{2}{N} \sum_{\mathbf{q} \in \mathcal{M}} \sin ^{2} \theta_{\mathbf{q}} .
$$

Therefore, Eq. (31) can be written in a more familiar form as a self-consistency equation for the gap parameter $\Delta$, using the definition of Eq. (32):

$$
\frac{2}{U}=\frac{2}{N} \sum_{\mathbf{q} \in \mathcal{M}} \frac{1}{\sqrt{\epsilon_{\mathbf{q}}^{2}+\Delta^{2}}}
$$

It is easy to show that $\Delta$ has the following smalland large- $U$ limiting behaviors for the $2 \mathrm{D}$ square lattice case:

$$
\Delta \longrightarrow \begin{cases}t e^{-2 \pi \sqrt{I I}}, & t \gg U \\ U / 2, & t \ll U .\end{cases}
$$

When these results are substituted into the groundstate energy $E_{0}$ and the excitation energy $E_{\mathbf{q}}$ of Eq. (34), we now obtain the correct limits in both the small- $U$ and large- $U$ cases. It is interesting to compare $E_{\mathbf{q}}$ of Eq. (34) with $\omega_{\mathbf{q}}$ of Eq. (27) where the gap parameter is simply given by $U / 2$. 


\section{A CCM ANALYSIS WITH MEAN-FIELD MODEL STATE}

A CCM analysis using $\left|\Phi_{M F}\right\rangle$ as the model state now becomes quite straightforward. As before, we write the ground-state function $\left|\Psi_{g}\right\rangle$ in the exponential form as

$$
\left|\Psi_{g}\right\rangle=e^{S}\left|\Phi_{M F}\right\rangle, \quad S=\sum_{n=1}^{N} S_{n},
$$

where $S_{n}$ are the $n$-body correlation operators consisting of the quasi-particle creation operators

$$
\begin{aligned}
S_{1} \equiv & \sum_{\mathbf{q} \in \mathcal{M}} \sum_{\sigma} S_{\mathbf{q}}^{\sigma} \alpha_{\mathbf{q} \sigma}^{+} \beta_{-\mathbf{q}-\sigma}^{+}, \\
S_{2} \equiv & \sum_{\mathbf{q}_{1}, \mathbf{q}_{2}, \mathbf{q}_{3}, \mathbf{q}_{4} \in \mathcal{M}}^{\prime}\left(S_{\mathbf{q}_{1} \mathbf{q}_{2} \mathbf{q}_{3} \mathbf{q}_{4}}^{(1)} \alpha_{\mathbf{q}_{1} \uparrow}^{+} \alpha_{\left.\mathbf{q}_{2}\right\rfloor}^{+} \beta_{\mathbf{q}_{3} \downarrow}^{+} \beta_{\mathbf{q}_{4} \downarrow}^{+}\right. \\
& +S_{\mathbf{q}_{1} \mathbf{q}_{2} \mathbf{q}_{3} \mathbf{q}_{4}}^{(2)} \alpha_{\mathbf{q}_{1} \uparrow}^{+} \alpha_{\mathbf{q}_{2} \uparrow}^{+} \beta_{\mathbf{q}_{3} \downarrow}^{+} \beta_{\mathbf{q}_{4} \downarrow}^{+} \\
& \left.+S_{\mathbf{q}_{1} \mathbf{q}_{2} \mathbf{q}_{3} \mathbf{q}_{4}}^{(3)} \alpha_{\mathbf{q}_{1} \downarrow}^{+} \alpha_{\mathbf{q}_{2} \downarrow}^{+} \beta_{\mathbf{q}_{3} \uparrow}^{+} \beta_{\mathbf{q}_{4} \uparrow}^{+}\right)
\end{aligned}
$$

etc. In Eq. (43), the prime on the summation implies the restriction $\mathbf{q}_{1}+\mathbf{q}_{2}+\mathbf{q}_{3}+\mathbf{q}_{4}=0$ due to the translational invariance of the lattice systems.

The charge excitation state (or the single-hole state) can be formulated in exactly the same fashion as before in Eqs. (23)-(26), but now using the quasiparticle operators instead of the original fermion operators.

\section{Conclusion}

In this short article, we outlined our formalism of the CCM for the Hubbard models on a bipartite lattice both at precisely half-filling and with a further single hole. Our aim has been to provide a CCM framework for a systematic investigation of the ground state and the single-hole state of halffilled Hubbard models, which is valid for a wide range of values of the interaction potential $U$.

For the Hubbard models on a bipartite lattice with a negative on-site potential $U$, which can be derived after consideration of the couplings with the lattice phonons, a similar CCM analysis can also be made. In particular, an obvious starting point at half-filling with negative $U$ is the so-called charge-density-wave state in which the $A$-sublattice is doubly occupied while the $B$-sublattice is empty. We intend to investigate these Hubbard models within the framework outlined in this article and intend to report our further results in the near future.

\section{ACKNOWLEDGMENTS}

We are grateful to J.B. Parkinson and N.J. Davidson for many useful discussions. One of us (R. F. B.) also gratefully acknowledges a research grant from the Science and Engineering Research Council (SERC) of Great Britain.

\section{References}

1. R. F. Bishop and H. Kümmel, Phys. Today 40(3), 52 (1987); R. F. Bishop, Theor. Chim. Acta 80, 95 (1991).

2. R. J. Bartlett, Theor. Chim. Acta 80, 71 (1991).

3. R. F. Bishop, J. B. Parkinson, and Y. Xian, Phys. Rev. B 43, 13782 (1991); Ibid. 44, 9425 (1991); Ibid. 46, 880 (1992); Ibid., J. Phys.: Condens. Matter 4, 5783 (1992); Ibid. 5, 9169 (1993).

4. R. F. Bishop, J. B. Parkinson, and Y. Xian, Theor. Chim. Acta 80, 181 (1991); R.F. Bishop and Y. Xian, Int. J. Quantum Chem.: Quantum Chem. Symp. 28 (1994).

5. R.F. Bishop, R.G. Hale, and Y. Xian, Phys. Rev. Lett. 73, 3157 (1994).

6. P. W. Anderson, Science 235, 1196 (1987); T. M. Rice and F. C. Zhang, Phys. Rev. B. 39, 815 (1989).

7. E. Manousakis, Rev. Mod. Phys. 63, 1 (1991); E. Dagotto, Rev. Mod. Phys. 66, 763 (1994).

8. M. Roger and J.H. Hetherington, Europhys. Lett. 11, 255 (1990); C. F. Lo, E. Manousakis, and Y. L. Wang, Phys. Lett. A 156, 42 (1991); F. Petit and M. Roger, Phys. Rev. B 49, 3453 (1994).

9. K. Emrich, Nucl. Phys. A 351, 379, 397 (1981).

10. J.R. Schrieffer, X.G. Wen, and S. C. Zhang, Phys. Rev. B 39, 11663 (1989). 